\title{
Troubles sexuels après curiethérapie par implants permanents d'I-125 dans le cancer localisé de prostate
}

\author{
Franck BLADOU1, Naji SALEM ${ }^{2}$, Gilles KARSENTY1, Gwenaelle GRAVIS ${ }^{3}$, Michelle SIMONIAN², \\ Roland ROSELLO4, Gérard SERMENT11.
}

\author{
1 Service d'Urologie, Hôpital Salvator, Marseille. ${ }^{2}$ Département de Radiothérapie ; 3 Département d'Oncologie \\ Médicale, ${ }^{4}$ Département de Radiologie, Institut Paoli-Calmettes, Marseille.
}

\section{RESUME}

La curiethérapie par implants permanents est une alternative à la prostatectomie radicale ou à l'irradiation externe en cas de cancer localisé de la prostate de bon pronostic. Les avantages de ce traitement sont une irradiation efficace mais précise, limitée à la glande prostatique avec une morbidité modérée et passagère. Les troubles de l'érection fréquents après chirurgie et irradiation externe sont retrouvés dans 6 à $61 \%$ des cas dans la littérature après curiethérapie. De tels écarts entre ces taux sont liés aux différences en terme de suivi, de définitions des troubles sexuels, d'instruments de mesure utilisés. Ces troubles de l'érection apparaissent entre 9 et 17 mois et seraient liés aux lésions radiques vasculaires des corps érectiles proches de l'apex prostatique (bulbe urétral et base des corps caverneux). Cependant, la majorité des troubles de l'érection répondent favorablement au traitements oraux tels que la yohimbine ou le sildénafil.

Parmi les options thérapeutiques à visée curative du cancer localisé de prostate, la curiethérapie par implants permanents est le traitement qui préserve le mieux la fonction érectile.

Mots clés : curietherapie, cancer de prostate, troubles de l'érection, qualité de vie

\section{INTRODUCTION}

Les troubles de l'érection représentent une complication fréquente après traitement des cancers localisés de prostate, malgré le développement de techniques chirurgicales permettant une préservation des bandelettes vasculo-nerveuses et la possibilité d'irradiation conformationnelle tridimensionnelle [1 - 8]. Depuis quelques années, des équipes ont développé la curiethérapie prostatique comme alternative aux traitements standards que sont la chirurgie et l'irradiation externe en cas de cancers précoces de bon pronostic (avec PSA $<10 \mathrm{ng} / \mathrm{ml}$, score de Gleason $<7$ et volume prostatique $<50 \mathrm{ml}$ ) $[9-11]$. Le renouveau des techniques de curiethérapie prostatique est lié à l'apport de l'échographie endorectale et aux nouvelles générations de logiciel de calculs de dosimétrie. Les équipes pionnières utilisant ces techniques publient des résultats à long terme qui semblent équivalents en terme de contrôle tumoral aux résultats rapportés après chirurgie dans les mêmes indications $[12,14]$. La faible morbidité à long terme de la curiethérapie représente son intérêt majeur et la forte attraction pour ce traitement de la part des patients ; de plus, elle est pratiquée le plus souvent dans le cadre d'une très courte hospitalisation ou en ambulatoire.

Dans des cas sélectionnés de bon pronostic, le choix thérapeutique (entre chirurgie, irradiation externe, curiethérapie ou surveillance) est de plus en plus déterminé non seu-

Correspondance :

PrFranck BLADOU - Service d'Urologie, Hôpital Salvator, 249 boulevard Sainte-Marguerite, B.P. 51, 13274 Marseille cedex 09 - Email franck.bladou@ap-hm.fr 
lement par le stade tumoral, l'âge du patient et l'existence de co-morbidité, mais également par les préférences du thérapeute et surtout du patient. En cas de choix thérapeutique éclairé, le patient devient un acteur décisif et décisionnel. Il peut, dans certains cas, choisir un traitement qui augmente ses chances de préserver une fonction sexuelle pour conserver une vie sexuelle active [15 - 17].

Les données de la littérature concernant les conséquences sexuelles de chaque option thérapeutique sont indispensables pour éclairer le patient dans son choix. Une méta-analyse récente [18] sur les taux de troubles de l'érection après traitements des cancers localisés de prostate rapporte les chiffres suivants : la probabilité de conserver une fonction érectile après curiethérapie est de 0,76 contre 0,60 après curiethérapie associée à une irradiation externe, 0,55 après irradiation, 0,34 après prostatectomie avec conservation des bandelettes vasculo-nerveuses et 0,25 après prostatectomie standard. Les critiques à apporter à ce travail concernent le nombre faible de patients traités par curiethérapie par rapport aux autres groupes thérapeutiques et l'absence de résultats après 2 ans de suivi pour ce dernier groupe. Les taux de troubles de l'érection rapportés dans la littérature sont de 6 à $84 \%$ après irradiation externe et de 6 à $61 \%$ après curiethérapie [19]. De telles différences entre les séries publiées sont à rattacher au caractère rétrospectif de la majorité des études, à l'utilisation de définitions différentes des troubles de l'érection et d'instruments de mesure souvent non validés. En effet, la puissance sexuelle est difficile à évaluer, il n'existe pas de définition unanime de la puissance sexuelle et les questionnaires utilisés n'étudient pas exactement les mêmes aspects de la sexualité et ne sont donc pas comparables. Avant la mise en route du traitement de leur cancer de prostate, tous les hommes n'ont pas la même qualité de vie sexuelle ni la même perception de leur puissance sexuelle. Ces éléments varient en fonction de l'âge, des antécédents médicaux (hypertension artérielle, diabète...) et des traitements médicamenteux dont les effets secondaires sur la sexualité sont possibles [19].

A la lecture des différentes études comparant les options thérapeutiques du cancer localisé de prostate, la curiethérapie semble être le traitement qui préserve le mieux la fonction érectile. L'analyse des scores de qualité de vie ne retrouve néanmoins pas de différence significative entre les différentes options à moyen terme [20, 21]. Les résultats sur les troubles de la sexualité en dehors de la fonction érectile restent très limités et incomplets, alors qu'ils représentent un reflet plus global et réel du vécu des patients.

\section{DEFINITION ET ANALYSE DE LA SEXUALITE}

Afin de pouvoir comparer des études et/ou des traitements entre eux, une définition commune des troubles sexuels est indispensable. La définition la plus consensuelle de l'impuissance sexuelle est celle du National Institutes of Health (NIH) aux Etats-Unis : c'est l'impossibilité réelle d'obtenir et de maintenir une érection suffisante pour permettre un rapport sexuel satisfaisant [22]. De nombreuses adaptations de cette définition sont utilisées dans l'analyse des résultats publiés, ce qui en rend l'interprétation plus incertaine. Par ailleurs, la sexualité masculine ne se limite pas à une capacité érectile. D'autres paramètres importants ne sont souvent pas analysés, notamment la libido, l'éjaculation, l'orgasme, la satisfaction de l'acte sexuel, l'intérêt sexuel, l'importance de la rigidité de l'érection... Les instruments de mesure des paramètres de la sexualité sont limités voire incomplets.

Depuis 1997, un questionnaire international validé dans de nombreuses langues (IIEF : International Index of Erectile Function) est disponible afin d'analyser les troubles de l'érection et de comparer différentes séries [23]. C'est un score de la fonction érectile d'origine anglo-saxonne auto administré qui n'évalue pas la qualité de la vie sexuelle de l'individu, il a uniquement pour but de quantifier le dysfonctionnement érectile. Il se compose de 5 items (le premier de 1 à 5 et les autres de 0 à 5 ), avec des scores allant de 1 à 25 . Les scores supérieurs à 21 traduisent une fonction érectile normale et les scores inférieurs à 21 traduisent des dysérections. Il ne présente pas d'intérêt dans son score moyen mais dans la comparaison des scores obtenus après différents traitements. Une analyse plus globale de la sexualité peut être proposée à partir de questionnaires rarement utilisés en pratique comme le Radiumhemmet scale of sexual functionning, le Brief Sexual Function Inventory (BSFI), le PR 25 de l'EORTC ou le Psychosocial Adjustment to Illness Scale (PAIS) qui incluent des questions sur le désir sexuel, la qualité de l'éjaculation, la satisfaction de la vie sexuelle, la fréquence d'érections spontanées, la fréquence des rapports sexuels [19]...

\section{ETIOLOGIE DES DYSFONCTIONS ERECTILES APRES CURIETHERAPIE}

L'étiologie des troubles de l'érection après traitement local curateur du cancer localisé de prostate est multifactorielle. Sont évoqués classiquement les lésions nerveuses, vasculaires, les traumatismes locaux, et le retentissement psychologique. La cause principale retenue de troubles de l'érection après prostatectomie radicale est le traumatisme ou la section des bandelettes vasculo-nerveuses [24]. Par contre, la dose d'irradiation délivrée aux bandelettes vasculo-nerveuses et analysée sur les dosimétries post-implantation ne semble pas être corrélée à la survenue de troubles de l'érection après curiethérapie [25, 26]. En fait, l'étiologie principale des troubles de l'érection après irradiation 
semble d'origine artérielle, par lésion radique des artérioles caverneuses $[27,28]$, mais peu d'études ont analysé les causes possibles après curiethérapie. Merrick a montré qu'une dose d'irradiation supérieure à 50 grays délivrée à l'urètre bulbaire et à la racine des corps caverneux était corrélée au taux de dysérection après curiethérapie [29, 30]. Il parait donc que le facteur principal des troubles de l'érection après curiethérapie soit plus l'atteinte radique de la vascularisation artérielle des corps caverneux et du bulbe urétral que l'atteinte nerveuse par irradiation des bandelettes vasculo-nerveuses. Ceci est corroboré par l'efficacité manifeste du sidénafil dans les dysérections après curiethérapie, cette molécule permettant une facilitation de l'ampliation des corps érectiles par dilatation des artérioles caverno-spongieuses [31].

A côté des causes organiques liées à l'irradiation, il est très peu fait état du rôle néfaste sur la libido de l'angoisse générée par le diagnostic de cancer de prostate, et le difficile choix thérapeutique, des conséquences organiques du traitement (troubles urinaires, ano-rectaux, douleur), du retentissement des traitements hormonaux parfois prescrits avant irradiation ou chirurgie [48], du retentissement socio-professionnel du diagnostic et du traitement, du regard et du soutien de l'entourage, de la partenaire sexuelle, de l'accompagnement par des professionnels psychooncologues, sexologues...

\section{INCIDENCE DES TROUBLES DE L'ERECTION APRES CURIETHERAPIE}

L'analyse des résultats de la littérature se heurte aux biais de la majorité des travaux publiés comme déjà évoqué : définition incertaine de la puissance sexuelle, analyses rétrospectives, sans analyses des co-morbidités, de l'état érectile des patients avant traitement... Les taux de troubles de l'érection après curiethérapie utilisée en monothérapie sont en majorité plus faible qu'après irradiation externe. Des biais de sélection doivent néanmoins être mentionnés car les patients qui choisissent ou qui sont orientés vers une curiethérapie ont souvent une tumeur de meilleur pronostic, sont souvent plus jeunes, avec un meilleur état de santé et une motivation plus importante pour préserver leur fonction érectile que les patients qui bénéficient d'une irradiation externe. Les résultats publiés de l'incidence des troubles de l'érection après curiethérapie varient de 6 à $61 \%$ et sont représentés dans le Tableau 1 .

Classiquement, la préservation des érections après implantation est corrélée avec la fonction érectile pré-thérapeutique, l'âge du patient, l'association d'une irradiation externe complémentaire et l'existence d'un diabète [43]. Le délai d'apparition des troubles de l'érection après curiethérapie peut varier de 9 mois en moyenne pour Merrick
[43] à 17 mois pour Zelefsky [13]. Dans la majorité des cas, le sildénafil est efficace et suffisant pour recréer des érections satisfaisantes après curiethérapie. Enfin, il existe une détérioration des érections avec un suivi plus long, notamment du fait du vieillissement des patients traités [45].

\section{TROUBLES DE L'EJACULATION ET AUTRES TROUBLES SEXUELS}

Les troubles de l'érection représentent un des aspects des troubles de la sexualité après traitements du cancer localisé de prostate, de loin le plus étudié. Pourtant, une détérioration de l'activité sexuelle peut être également liée à des troubles de l'éjaculation, de la libido, des sensations orgasmiques... Les troubles de l'éjaculation à distance de la curiethérapie sont retrouvés dans 7 à $45 \%$ des études, et consistent en une diminution plus ou moins importante du volume de l'éjaculât et de son aspect (généralement plus fluide et transparent), une gène ou douleur lors de l'éjaculation dans 3 à $11 \%$ des cas, et une hémospermie fréquente les semaines après la curiethérapie et rarement persistante $[19,32,46,47]$.

Une analyse plus détaillée des paramètres sexuels autres que la fonction érectile mériterait d'être systématiquement faite au cours des études sur le retentissement sexuel des traitements du cancer de prostate et notamment de la curiethérapie.

\section{PRISE EN CHARGE DES TROUBLES SEXUELS APRES CURIETHERAPIE}

La majorité des patients qui présentent des troubles de l'érection après curiethérapie répondent favorablement au sildénafil [31]. Dans un travail récent, Merrick [43] retrouve $85 \%$ d'efficacité dans cette indication. La réponse au sildénafil était corrélée au potentiel érectile pré-implantation. Chez les patients qui présentaient un score IIEF de 2 avant traitement (score 2 = érections toujours ou presque toujours suffisantes pour une pénétration vaginale), l'efficacité du sildénafil était de $95 \%$, contre $70 \%$ chez les patients qui avaient un score pré-thérapeutique de 1 (score $1=$ érections suffisantes pour une pénétration vaginale mais considérées comme insuffisantes).

Dans notre expérience, avec un suivi d'au moins 1 an après implantation, 89 patients sur $260(34,2 \%)$ se sont plaints de troubles de l'érection induits par le traitement, à type de troubles de l'obtention et/ou du maintien des érections chez 77 patients $(29,6 \%)$ et d'impuissance chez 12 patients $(4,6 \%)$. Ces patients ont eu un traitement pour améliorer les érections selon l'algorithme thérapeutique suivant : première prescription : yohimbine ${ }^{\circledR}$ pendant 3 mois, si échec, 
Tableau 1 : Incidence des troubles de l'érection après curiethérapie pour cancer de prostate.

\begin{tabular}{|c|c|c|c|c|c|c|c|}
\hline$\overline{\text { Auteurs }}$ & Isotope & RTE & $\begin{array}{l}\text { Patients } \\
\text { n }\end{array}$ & $\begin{array}{l}\text { Age } \\
\text { moyen }\end{array}$ & $\begin{array}{l}\text { Erections pré- } \\
\text { curie }(\%)\end{array}$ & $\begin{array}{l}\text { Suivi } \\
\text { moyen }\end{array}$ & $\begin{array}{c}\text { Incidence } \\
(\%)\end{array}$ \\
\hline Blasko, 1993 [10] & $\mathrm{I} / \mathrm{Pd}$ & + & 469 & nd & nd & 38 & $\begin{array}{l}<70 \text { ans : } 15 \\
>70 \text { ans }: 50\end{array}$ \\
\hline Kleinberg, 1994 [32] & I & - & 31 & 67 & 58 & $>12$ & 6 \\
\hline Wallner, 1994 [33] & $\mathrm{I}$ & - & 62 & 67 & 61 & 19 & 19 \\
\hline Kaye, 1995 [11] & I & + & 72 & 71 & 61 & 26 & 25 \\
\hline Stone, 1995 [34] & $\mathrm{I} / \mathrm{Pd}$ & - & 58 & 68 & 21 & 12 & 8 \\
\hline Chaikin, 1996 [35] & $\mathrm{I} / \mathrm{Pd}$ & - & 37 & nd & 73 & 18 & 45 \\
\hline Stock, 1996 [36] & $\mathrm{I} / \mathrm{Pd}$ & - & 97 & 69 & 66 & 18 & 21 \\
\hline Arterbery, 1997 [37] & $\mathrm{I} / \mathrm{Pd}$ & - & 51 & nd & 69 & nd & 13 \\
\hline Koutrouvelis, 1998 [38] & $\mathrm{I} / \mathrm{Pd}$ & - & 130 & 71 & nd & nd & 5 \\
\hline Sharkey, 1998 [39] & $\mathbf{P d}$ & - & 434 & 73 & nd & 28 & 15 \\
\hline Zelefsky, 1999 [13] & I & - & 145 & 64 & 88 & 24 & 21 \\
\hline Sanchez-Ortiz, 2000 [40] & $\mathrm{I} / \mathrm{Pd}$ & - & 114 & 69 & 71 & 23 & 51 \\
\hline Sharkey, $2000[41]$ & $\mathrm{Pd}$ & - & 299 & 73 & nd & nd & 15 \\
\hline Zelefsky, 2000 [14] & I & - & 248 & 65 & 89 & 48 & 29 \\
\hline Stock, 2001 [42] & $\mathrm{I} / \mathrm{Pd}$ & - & 416 & 66 & 75 & 32 & $\begin{array}{l}21 \text { à } 3 \text { ans } \\
41 \text { à } 6 \text { ans }\end{array}$ \\
\hline Merrick, 2002 [43] & $\mathrm{I} / \mathrm{Pd}$ & - & 181 & 65 & 100 & 40 & 61 \\
\hline Bladou, 2003 [44] & I & - & 260 & 65 & nd & 12 & 34 \\
\hline
\end{tabular}


prescription de viagra ${ }^{\circledR}$ et si échec, prescription d'injections intra-caverneuses. Les prescriptions ont été les suivantes : 36 patients $(40,4 \%)$ sont équilibrés avec yohimbine ${ }^{\circledR}, 41$ patients $(46 \%)$ avec viagra ${ }^{\circledR}$ et 12 patients $(13,4 \%)$ avec injection intra-caverneuse.

Trente neuf parmi les 89 patients $(43,8 \%)$ qui ont présenté des troubles de l'érection avaient eu un blocage androgénique avant l'implantation [44].

\section{CONCLUSION}

Les troubles de l'érection après curiethérapie pour cancer localisé de prostate sont retrouvés dans 6 à $61 \%$ des séries de la littérature. Les différences notables entre les publications sont à mettre sur le compte des différences en terme de suivi, de définitions des troubles de l'érection, des techniques de mesure de ces troubles (par le chirurgien, par auto-questionnaire, avec des instruments validés ou non...). L'apparition des troubles de l'érection après curiethérapie est corrélée à plusieurs facteurs tels que l'âge du patient, l'état érectile pré-thérapeutique, l'association à un traitement hormonal ou une irradiation externe, l'existence de tares telles que le diabète. L'irradiation du bulbe urétral et de la base des corps érectiles semble à l'origine de lésions occlusives des artérioles bulbo-caverneuses. Cette atteinte vasculaire provoquerait des troubles de l'érection qui ne seraient pas liés à une atteinte des bandelettes vasculo-nerveuses. A l'inverse des patients après prostatectomie radicale, la majorité des troubles de l'érection répondent favorablement au sildénafil.

Parmi les options thérapeutiques à visée curative du cancer localisé de prostate, la curiethérapie par implants permanents d'iode 125 ou de palladium 103 est le traitement qui préserve le mieux la fonction érectile avec des troubles de l'érection induits qui répondent le plus favorablement au sildénafil.

\section{REFERENCES}

1. McCAMMON K.A., KOLM P., MAIN B. et al. : Comparative quality-of-life analysis after radical prostatectomy or external beam radiation for localized prostate cancer. Urology, 1999, 54 : 509-516.

2. BANKER F.L. : The preservation of potency after external beam irradiation for prostate cancer. Int. J. Radiat. Oncol. Biol. Phys., 1988, $15: 219-220$.

3. MARTINEZ A, GONZALEZ J, STROMBERG J, et al. : Conformal prostate brachytherapy: Initial experience of a phase I/II dose-escalating trial. Int. J. Radiat. Oncol. Biol. Phys., 1995, 33 : 1019-1027.

4. LILLEBY W., FOSSA S.D., WAEHRE H.R, et al. : Long-term morbidity and quality of life in patients with localized prostate cancer undergoing definitive radiotherapy or radical prostatectomy. Int. J. Radiat. Oncol. Biol. Phys., 1999, 43 : 735-743.

5. LITWIN M.S., FLANDERS S.C., PASTA D.J. et al. : Sexual function and bother after radical prostatectomy or radiation for prostate cancer : Multivariate quality-of-life analysis from CaPSURE. Urology, 1999, 54 : 503-508.

6. TURNER S.L., ADAMS K., BULL C.A. et al. : Sexual dysfunction after radical radiation therapy for prostate cancer : A prospective evaluation. Urology, 1999, 54 : 124-129.

7. STANFORD J.L., FENG Z., HAMILTON A.S. et al. : Urinary and sexual function after radical prostatectomy for clinically localized prostate cancer : The Prostate Cancer Outcomes Study. J. Am. Med. Ass., 2000, $283:$ 354-360.

8. CATALONA W.J., CARVALHAL G.F., MAGER D.E. et al. : Potency, continence and complication rates in 1,870 consecutive radical retropubic prostatectomies. J. Urol., 1999, 162 : 433438.

9. WHITMORE W.F. Jr, HILARIS B., GRABSTALD H. : Retropubic implantation of iodine 125 in the treatment of prostatic cancer. J. Urol., 1972, 108 : 918-920.

10. BLASKO J.C., GRIMM P.D., RAGDE H. : Brachytherapy and organ preservation in the management of carcinoma of the prostate. Semin. Radiat. Oncol., 1993, 3 : 240-249.

11. KAYE K.W., OLSON D.J., PAYNE J.T. : Detailed preliminary analysis of iodine-125 implantation for localized prostate cancer using percutaneous approach. J. Urol., 1995, 153 : 1020-1025.

12. RAGDE H., BLASKO J.C., GRIMM P.D. et al. : Interstitial iodine-125 radiation without adjuvant therapy in the treatment of clinically localized prostate cancer. Cancer, 1997, $80: 442-$ 453.

13. ZELEFSKY M.J., WALLNER K.E., LING C.C. et al. : Comparison of the 5-year outcome and morbidity of three-dimensional conformal radiotherapy versus transperineal permanent iodine125 implantation for early-stage prostatic cancer. J. Clin. Oncol., 1999, $17: 517-522$.

14. ZELEFSKY M.J., HOLLISTER T., RABEN A. et al. : Five-year biochemical outcome and toxicity with transperineal CT-planned permanent I- 125 prostate implantation for patients with localized prostate cancer. Int. J. Radiat. Oncol. Biol. Phys., 2000, $47: 1261-1266$.

15. BRODY D.S. : The patient's role in clinical decision-making. Ann. Intern. Med., 1980, 93 : 718-722.

16. QUILL T.E. : Partnerships in patient care : a contractual approach. Ann. Intern. Med., 1983, 98 : 228-234.

17. BROCK D.W., WARTMAN S.A. : When competent patients make irrational choices. N. Engl. J. Med., 1990, 322 :15951599.

18. ROBINSON J.W., PSYCH C., MORITZ S. et al. : Meta-analysis of rates of erectile function after treatment of localized prostate carcinoma. Int. J. Radiat. Oncol. Biol. Phys., 2002, 54 : 1063-1068.

19. INCROCCI L., KOOS SLOB A., LEVENDAG P.C. Sexual (dys)function after radiotherapy for prostate cancer : a review. Int. J. Radiat. Oncol. Biol. Phys., 2002, 52 : 681-693.

20. LITWIN M.S., HAYS R.D., FINK A. et al. : Quality-of-life outcome in men treated for localized prostate cancer. J. Am. Med. Ass., 1995, 273 : 129-135. 
21. LITWIN M.S. : Health-related quality-of-life after treatment for localized prostate cancer. Cancer, 1995, $75: 2000-2003$.

22. NIH consensus development panel on impotence. J. Am. Med. Ass., 1993, $270: 83-90$.

23. ROSEN R.C. RILEY A., WAGNER G. et al. : The International Index of Erectile Function (IIEF). A multidimensional scale for assessment of erectile dysfunction. Urology, 1997, $49: 822-830$.

24. IEPOR H., GREGERMAN M., CROSBY R. et al. : Precise localization of the autonomic nerves from the pelvic plexus to the corpora cavernosa : A detailed anatomical study of the adult male pelvis. J. Urol., 1985, 133 : 207-212.

25. DI BIASE S.J., WALLNER K., TRALINS K. et al. ; Brachytherapy radiation doses to the neurovascular bundles. Int. J. Radiat. Oncol. Biol. Phys., 2000, 46 : 1301-1307.

26. MERRICK G.S., BUTLER W.M., DORSEY A.T. et al. : A comparison of radiation dose to the neurovascular bundles in men with and without prostate brachytherapy-induced erectile dysfunction. Int. J. Radiat. Oncol. Biol. Phys., 2000, 48 : 10691074.

27. GOLDSTEIN I., FELDMAN M.I., DECKERS P.J. et al. : Radiation-associated impotence. J. Am. Med. Ass., 1984, 251 : 903-910.

28. ZELEFSKY M.J., EID J.F. : Elucidating the etiology of erectile dysfunction after definitive therapy for prostatic cancer. Int. J. Radiat. Oncol. Biol. Phys., 1998, 40 : 129-133.

29. MERRICK G.S., WALLNER K., BUTLER W.M. et al. : A comparison of radiation dose to the bulb of the penis in men with and without prostate brachytherapy-induced erectile dysfunction. Int. J. Radiat. Oncol. Biol. Phys., 2001, 50 : 597-604.

30. MERRICK G.S., BUTLER W.M., WALLNER K.E. et al. : The importance of radiation doses to the penile bulb vs. crura in the development of postbrachytherapy erectile dyfunction. Int. J. Radiat. Oncol. Biol. Phys., 2002, 54 : 1055-1062.

31. MERRICK G.S., BUTLER W.M., LIEF J.H. et al. : Efficacy of sildenafil citrate in prostate brachytherapy patients with erectile dysfunction. Urology, 1999, 53 : 1112-1116.

32. KLEINBERG L., WALLNER K., ROY J. et al. : Treatmentrelated symptoms during the first year following transperineal I125 prostate implantation. Int. J. Radiat. Oncol. Biol. Phys., 1994, 28 : 985-990.

33. WALLNER K., ROY J., ZELEFSKY M. et al. : Short-term freedom from disease progression after I-125 prostate implantation. Int. J. Radiat. Oncol. Biol. Phys., 1994, 30 : 405-409.

34. STONE N.N, RAMIN S.A., WESSON M.F. et al. : Laparoscopic pelvic lymph node dissection combined with real-time interactive transrectal ultrasound guided transperineal radioactive seed implantation of the prostate. J. Urol., 1995, 153 : 15551560.

35. CHAIKIN D.C., BRODERICK G.A., MALLOY T.R. et al. : Exectile dysfunction following minimally invasive treatments for prostate cancer. Urology, 1996, 48 : 100-104.

36. STOCK R.G., STONE N.N., DE WYNGAERT J.K. et al. : Prostate specific antigen findings and biopsy results following interactive ultrasound guided transperineal brachytherapy for early stage prostate carcinoma. Cancer, 1996, 77 : 2386-2392.
37. ARTERBERY V.E., FRAZIER A., DALMIA P. et al. : Quality of life after permanent prostate implant. Surg. Oncol., 1997, 13 : 461-464.

38. KOUTROUVELIS P.G. : Three-dimensional stereotactic posterior ischiorectal space computerized tomography guided brachytherapy of prostate cancer : A preliminary report. J. Urol., 1998, $159: 142-145$.

39. SHARKEY J., CHOVNICK S.D., BEHAR R.J. et al. : Outpatient ultrasound-guided palladium-103 brachytherapy for localized adenocarcinoma of the prostate : A preliminary report of 434 patients. Urology, 1998, $51: 796-803$.

40. SANCHEZ-ORTIZ R.F0, BRODERICK G.A., ROVNER E.S. et al. : Erectile function and quality of life after interstitial radiation therapy for prostate cancer. Int. J. Impotence Res., 2000, 12 (Suppl. 3) : 18-24.

41. SHARKEY J., CHOVNICK S.D., BEHAR RJ. et al. : Minimally invasive treatment for localized adenocarcinoma of the prostate: Review of 1048 patients treated with ultrasound-guided palladium-103 brachytherapy. J. Endourol., 2000, 14 : 343-350.

42. STOCK R.G., KAO J., STONE N.N. : Penile erectile function after permanent radioactive seed implantation for treatment of prostate cancer. J. Urol., 2001, $165: 436-439$.

43. MERRICK G.S., BUTLER W.M., GALBREATH R.W. et al. : Erectile function after permanent prostate brachytherapy. Int. J. Radiat. Oncol. Biol. Phys., 2002, 52 : 893-902.

44. BLADOU F., SALEM N., SIMONIAN M. et al. : La curiethérapie par implants permanents d'iode 125 dans le cancer localisé de prostate : résultats à 4 ans. Soumis à Prog. Urol.

45. FELDMAN H.A., GOLDSTEIN .I, HATZICHRIFTOU D.G. et al. : Impotence and its medical and psychosocial correlates: Results of the Massachusetts Male Aging Study. J. Urol., 1994, $151: 54-61$.

46. FOWLER J.E. Jr, BARZELL W., HILARIS B.S. et al. : Complications of iodine-125 implantation and pelvic lymphadenectomy in the treatment of prostatic cancer. J. Urol., 1979, 121 : 447451

47. ARTERBERY V.E., WALLNER K., ROY J. et al. : Short-term morbidity from CT-planned transperineal I-125 prostate implants. Int. J. Radiat. Oncol. Biol. Phys., 1993, 25 : 661-667.

48. POTTERS L., TORRE T., FEARN P.A. et al. : Potency after permanent prostate brachytherapy for localized prostate cancer. Int. J. Radiat. Oncol. Biol. Phys., 2001, 50 : 1235-1242. 


\begin{abstract}
Sexual disorders after brachytherapy by permanent l-125 implants in localized prostate cancer

Franck BLADOU, Naji SALEM, Gilles KARSENTY, Gwenaelle GRAVIS, Michelle SIMONIAN, Roland ROSEL-

LO, Gérard SERMENT.
\end{abstract}

Brachytherapy by permanent implants is an alternative to radical prostatectomy or external beam radiotherapy for good prognosis localized prostate cancer. The advantages of this treatment are effective and precise irradiation, limited to the prostate gland with moderate and transient morbidity. Erectile dysfunction, frequent erection after surgery and external beam radiotherapy, is observed in $6 \%$ to $61 \%$ of cases in the literature after brachytherapy. This wide range is related to differences in terms of follow-up, definition of sexual disorders, and the measuring instruments used. These erectile disorders occur between 9 and 17 months after treatment and appear to be related to vascular radiation lesions of the erectile bodies close to the prostatic apex (urethral bulb and base of the corpora cavernosa). However, the majority of erectile disorders respond favourably to oral treatments such as yohimbine or sildenafil.

Among the various curative treatment options for localized prostate cancer, permanent implant brachytherapy is the treatment ensuring the best preservation of erectile function.

Key-Words: brachytherapy, prostate cancer, erectile dysfunction, quality of life 